\title{
Noise caused by semiconductor lasers in high-speed fiber-optic links
}

\author{
Olsen, C. M.; Stubkjær, Kristian; Olesen, H.
}

Published in:

Journal of Lightwave Technology

Link to article, DOI:

10.1109/50.19092

Publication date:

1989

Document Version

Publisher's PDF, also known as Version of record

Link back to DTU Orbit

Citation (APA):

Olsen, C. M., Stubkjær, K., \& Olesen, H. (1989). Noise caused by semiconductor lasers in high-speed fiber-optic links. Journal of Lightwave Technology, 7(4), 657-665. https://doi.org/10.1109/50.19092

\section{General rights}

Copyright and moral rights for the publications made accessible in the public portal are retained by the authors and/or other copyright owners and it is a condition of accessing publications that users recognise and abide by the legal requirements associated with these rights.

- Users may download and print one copy of any publication from the public portal for the purpose of private study or research.

- You may not further distribute the material or use it for any profit-making activity or commercial gain

- You may freely distribute the URL identifying the publication in the public portal

If you believe that this document breaches copyright please contact us providing details, and we will remove access to the work immediately and investigate your claim. 


\title{
Noise Caused by Semiconductor Lasers in High- Speed Fiber-Optic Links
}

\author{
C. M. OLSEN, K. E. STUBKJAER, Member, IEeE, AND H. OLESEN
}

\begin{abstract}
We present theoretical and experimental results for the signal-to-noise $(S / N)$ ratio caused by mode partition noise, intensity noise, and reflection-induced noise in optical data links. Under given conditions an additional noise source with a $S / N$ ratio of $20 \mathrm{~dB}$ will cause a power penalty of $1 \mathrm{~dB}$ in order to maintain a $10^{-9}$ bit error rate. From our numerical simulations we predict the maximum allowable dispersion in the presence of mode partition noise to be approximately $\mathbf{4 0}$ percent of a clock period. This figure is almost independent of bit rate and laser structure and agrees well with our measurements and with results of other workers. Numerical simulations of a BH and a TJS laser were carried out at four bit rates from $565 \mathrm{Mbit} / \mathrm{s}$ to 4.5 Gbit/s and the measurements were done at $2.2 \mathrm{Gbit} / \mathrm{s}$ using a TJS laser.
\end{abstract}

\section{INTRODUCTION}

$\mathrm{H}$ IGH SPEED optical fiber transmission systems are of interest for long-haul applications such as trunk lines and undersea cable transmission, and for fast optical data links in computer networks. In both cases mode partition noise [1]-[3], chirping [4], and reflection-induced noise [5]-[6] have been identified as factors limiting the transmission distance. Data links have special requirements such as extremely low bit error rate (BER) of $10^{-12}$ or lower, inexpensive components, and many connectors in the transmission path [7]. One may therefore consider using $\mathrm{AlGaAs}$ lasers and multimode fibers or using singlemode fibers at wavelengths where the dispersion is significant. Even if InGaAsP lasers are used (at 1.3 or 1.55 $\mu \mathrm{m}$ ) dispersion may still be a limiting factor in connection with mode partition noise or chirping.

Mode partition noise arises due to random power fluctuations among longitudinal modes in a laser [3]. The fluctuations in the main and side modes are anticorrelated so that the total output fluctuations are significantly reduced. However, when laser light distributed on several modes is transmitted through a dispersive fiber, the signals emitted in different modes will be delayed relative to each other, and the fluctuations on the total output signal will increase with increasing dispersion because the anti-

Manuscript received March 29, 1988; revised July 27, 1988. This work was partially supported by IBM Research, Yorktown Heights, NY. Travel support was provided by J. Teglh $\phi$ i of IBM Denmark.

C. M. Olsen was with Electromagnetics Institute, Technical University of Denmark, DK-2800 Lyngby, Denmark. He is now with NKT Elektronik, DK-2600 Glostrup, Denmark.

K. E. Stubkjaer is with the Electromagnetics Institute, Technical University of Denmark, DK-2800 Lyngby, Denmark.

H. Olesen is with TFL Telecommunications Research Laboratory, DK2970 H $\phi$ rsholm, Denmark. correlation is destroyed [8]. In addition, mode partition noise will cause timing jitter at the receiver for high bit rates, and pulse broadening will occur due to the effective low-pass filtering of the fiber and the receiver.

In an earlier paper [3] we presented a numerical model for simulation of mode partition noise in lasers under $\mathrm{CW}$ and pulsed operation. Here, we will describe how the model can be extended to include the effect of fiber dispersion and receiver low-pass filtering in order to predict the signal-to-noise $(S / N)$ ratio and power penalty in a practical system. We will also present measurements of the $S / N$ ratio under various experimental conditions.

The paper is organized as follows: in Section II we give a brief summary of the theoretical model of mode partition noise, and Section III contains a description of the low-pass filtering and ways to calculate the $S / N$ ratio and Ogawa's $k$-factor. In Section IV we give several numerical results for noise statistics, $S / N$ ratio, and timing jitter. The simulations are carried out for an $880-\mathrm{nm}$ TJS laser and a 1300-nm BH laser. Finally, Section V presents the experimental results on $S / N$ ratio.

\section{THEORY}

The numerical model for prediction of the $S / N$ ratio and the jitter due to laser noise is based on the multimode rate equations with Langevin noise terms as described in [3]:

$$
\begin{aligned}
& \frac{d N}{d t}=J-R(N)-\sum_{i} \Gamma G_{i}(N) S_{i}+F_{N}(t) \\
& \frac{d S_{i}}{d t}=\left[\Gamma G_{i}(N)-\frac{1}{\tau_{p}}\right] S_{i}+\beta_{i} B N\left(N+P_{0}\right)+F_{i}(t)
\end{aligned}
$$

where $N$ is the carrier number, $P_{0}$ is the hole number without injection and is approximately equal to the number of acceptors, and $S_{i}$ is the photon number in the $i$ th longitudinal mode. $J$ is the pumping in electrons per second $R(N)$ the total spontaneous recombination rate, $\Gamma$ the mode confinement factor, $\tau_{p}$ the photon lifetime, $\beta_{i}$ the fraction of spontaneous emission coupled into mode $i$, and $B$ the band-to-band recombination coefficient. The Langevin noise terms $F_{N}(t)$ and $F_{i}(t)$ are implemented by generating a set of jointly Gaussian random numbers as described in detail in [3]. This procedure is valid for the typical time step of 5-10 ps. 
The gain $G_{i}(N)$ is assumed to have a parabolic wavelength dependence but contrary to [3] the wavelength shift of the gain maximum $\lambda_{0}(N)$ with carrier number (i.e., the band filling effect) is also taken into account. The nonlinear gain is implemented in a simplified form [9] and with the usual linear dependence on the carrier number the gain is given by

$$
\begin{aligned}
G_{i}(N)= & G_{N}\left(N-N_{0}\right) \\
& \cdot\left\{1-2\left(\frac{\lambda_{i}-\lambda_{0}(N)}{\Delta \lambda_{G}}\right)^{2}\right\}\left(1-\epsilon S_{\mathrm{tot}}\right)
\end{aligned}
$$

where $G_{N}$ is a gain coefficient, $N_{0}$ the carrier number at transparency, $\lambda_{i}$ the wavelength of the $i$ th mode and $\Delta \lambda_{G}$ the width of the gain curve (FWHM). We have assumed homogeneous broadening and a constant width of the gain curve. $\epsilon$ is the nonlinear gain coefficient [9] and $S_{\text {tot }}=\Sigma S_{i}$ the total photon number. The recombination rate $R(N)$ is taken as [10]

$$
R(N)=A N+B N\left(N+P_{0}\right)+C N\left(N+P_{0}\right)^{2}
$$

where $A, B$, and $C$ are constants. The Auger recombination represented by the third term is negligible for $\mathrm{Ga}$ AlAs lasers.

The coupled rate equations (1)-(2) can be solved numerically to give the time variation of carrier and photon numbers under specified operating conditions. The model has been tested by simulating laser noise under CW operation as well as large signal pulsed operation. It was found that the resulting noise levels are in agreement with those found theoretically and experimentally by other workers [3], [6], [8]. Optical feedback is not included in (1)-(2). Development of a multimode noise model which for all modes can account for the amplitude and phase of the light coupled back from an external reflection is a complicated task which is left for future work.

\section{Simulation of Digital Modulation}

The model has been used to simulate noise properties of semiconductor lasers modulated with binary sequences at bit rates from $565 \mathrm{Mbit} / \mathrm{s}$ to $4.5 \mathrm{Gbit} / \mathrm{s}$. The resulting $S / N$ ratio for the total output signal from the laser is obtained after summation of the signals from the individual modes $\Sigma S_{i}(t)$.

Strictly speaking, the fiber is linear in amplitude rather than intensity, but for our purpose it is sufficient to treat a dispersive fiber as a number of delay lines, one for each laser mode. We can therefore calculate the output from the fiber by delaying the time responses of the individual modes by their relative propagation delay $\Delta \tau_{i}$, before summation: $\Sigma S_{i}\left(t-\Delta \tau_{i}\right)$. This is equivalent to a lowpass filter with the following "transfer function":

$$
H_{f}(f)=\sum_{i}\left\langle a_{i}\right\rangle e^{-j 2 \pi f \Delta r_{i}}
$$

where $a_{i}=S_{i} / \Sigma S_{i}$ is the normalized photon number in mode $i$. The relative delay $\Delta \tau_{i}$ can be expressed as $i \cdot \lambda_{d}$ - $\delta \cdot L$ where $\lambda_{d}$ is the mode spacing of the laser, $\delta$ is the dispersion coefficient, and $L$ is the fiber length. However, (5) is not a true transfer function because it depends on the mode spectrum of the laser.

The receiver low-pass filtering is represented by a third order Bessel filter with the following transfer function:

$$
\begin{gathered}
H_{B}(f)=\left\{1-\frac{2}{5}\left(\frac{f}{f_{0}^{\prime}}\right)^{2}+j\left(\frac{f}{f_{0}^{\prime}}-\frac{1}{15}\left(\frac{f}{f_{0}^{\prime}}\right)^{3}\right)\right\}^{-1}, \\
f_{0}^{\prime}=0.57 f_{0}
\end{gathered}
$$

where $f_{0}$ corresponds to the $3-\mathrm{dB}$ cutoff which is taken as half of the bit frequency in all the simulations which are presented here. The transfer function of the filter is shown in Fig. 1 together with an example of the transfer function for the fiber $H_{f}(f)$ taken for a fiber dispersion of 0.12 $\mathrm{ns} / \mathrm{nm}$ and the laser spectrum in Fig. 6(a). Also the total transfer function for fiber and filter is shown in Fig. 1.

The filtered time response is sampled at the position in the time slot where the response for marks on average has its maximum. The resulting signal-to-noise ratio $S / N$ is calculated from [11]:

$$
S / N=\frac{\left(b_{1}-b_{0}\right)^{2}}{\left(\sigma_{1}+\sigma_{0}\right)^{2}}
$$

where $b_{1}$ and $b_{0}$ are the mean values and $\sigma_{1}$ and $\sigma_{0}$ are the standard deviations of the sampled amplitudes for transmitted marks and spaces, respectively. The simulations included 11 longitudinal modes with the weakest side modes carrying only 1-2 percent of the total output power.

Assuming that the sampled amplitudes of the signal follow a Gaussian distribution, the system penalty $P_{a}$ (in optical decibels) due to the laser noise (or other noise sources) can be calculated as [12]

$$
P_{a}=5 \log \left\{(S / N) /\left((S / N)-Q^{2}\right)\right\}
$$

where $Q$ is related to the bit error rate BER:

$$
\mathrm{BER}=\sqrt{\frac{1}{2 \pi}} \int_{Q}^{\infty} \exp \left(-\frac{x^{2}}{2}\right) d x .
$$

In (9) equal probability for marks and spaces is assumed. $P_{a}$ is a useful parameter which gives the required increase in received power to maintain the bit error rate when an extra noise source is "switched on." For a BER of $10^{-9}$, penalties of 0.1 and $1 \mathrm{~dB}$ result for $S / N$ ratios of 29 and $20 \mathrm{~dB}$, respectively.

Mode partition in semiconductor lasers is commonly described by Ogawa's $k$-factor [1]. It is assumed that the total photon number of all lasing modes is constant (although the individual modes are fluctuating), and that spontaneous emission can be neglected for the weaker side modes. Although these assumptions are known not to be valid in practice, the $k$-factor still seems to provide a good measure. The definition is the following:

$$
k^{2}=\frac{\sum_{i}\left[\left\langle a_{i}^{2}\right\rangle-\left\langle a_{i}\right\rangle^{2}\right]}{\sum_{i}\left(\left\langle a_{i}\right\rangle-\left\langle a_{i}\right\rangle^{2}\right)}
$$




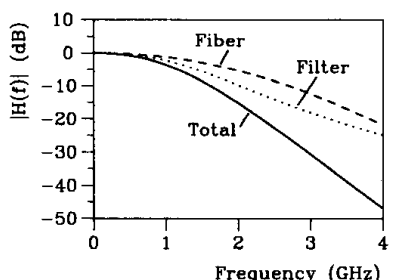

Fig. 1. Frequency transfer function for the third order Bessel filter which is used in the simulations $\left(B_{0}=2.24 \mathrm{Gbit} / \mathrm{s}\right)$, for the fiber assuming a total dispersion of $0.12 \mathrm{~ns} / \mathrm{nm}$ and the spectrum in Fig. 6(a), and the total transfer function of fiber and filter.

which is based on all the modes. Alternatively, an individual $k$-factor can be defined for each mode:

$$
k_{i}^{2}=\frac{\left\langle a_{i}^{2}\right\rangle-\left\langle a_{i}\right\rangle^{2}}{\left\langle a_{i}\right\rangle-\left\langle a_{i}\right\rangle^{2}}=\frac{\left\langle a_{i}^{2}\right\rangle-\left\langle a_{i}\right\rangle^{2}}{\left\langle a_{i}\right\rangle^{2}} \frac{\left\langle a_{i}\right\rangle}{1-\left\langle a_{i}\right\rangle} .
$$

The individual $k$-factors will be smaller for the weaker side modes than for the central modes because of the increasing amount of spontaneous emission, and therefore $k_{i}$ will in general be different from $k$ [1]. As shown in (11), $k_{i}^{2}$ is expressed as the inverse signal-to-noise ratio in mode number $i$, multiplied by the ratio between the average power in mode $i$ and the total average power in all the other modes. Both $k$ and $k_{i}$ will lie between 0 and 1 with larger values indicating stronger mode partition [1].

Ogawa also derived an expression for the signal-tonoise due to mode partition after transmission through a dispersive fiber [12]. It is given by

$$
\begin{aligned}
\frac{S}{N}= & {\left[k ^ { 2 } \frac { ( \pi B _ { 0 } ) ^ { 4 } } { 4 } \left(\sum_{i}\left(\Delta \tau_{i}\right)^{4}\left\langle a_{i}\right\rangle\right.\right.} \\
& \left.\left.-\left(\sum_{i}\left(\Delta \tau_{i}\right)^{2}\left\langle a_{i}\right\rangle\right)^{2}\right)\right]^{-1}
\end{aligned}
$$

where $B_{0}$ is the bit rate and $\Delta \tau_{i}$ the relative delay as introduced in (5). It should be emphasized that (12) is only valid for $\Delta \tau_{i} B_{0} \ll 1$ since it relies on the assumption of a parabolic waveform in the vicinity of the sampling time. In practice, several other factors contribute to reducing the $S / N$ ratio, e.g., the intensity noise of the laser (fluctuations in the total output) and the reflection-induced noise. We shall use (12) in the following sections to compare with numerical simulations and experiments.

\section{Numerical Results}

The model described in the previous sections was used to estimate the signal-to-noise ratio $S / N$ which is caused by laser noise in a PCM system with a dispersive fiber. Simulations for both a $1300-\mathrm{nm}$ BH laser and an $880-\mathrm{nm}$ TJS laser were carried out. The 1300-nm laser is of interest for both local network and for long haul communication links and the 880-nm laser is considered to be of interest to local network applications. The laser parameters listed in Table I were used in the simulations.

Fig. 2 gives an example of the calculated response for
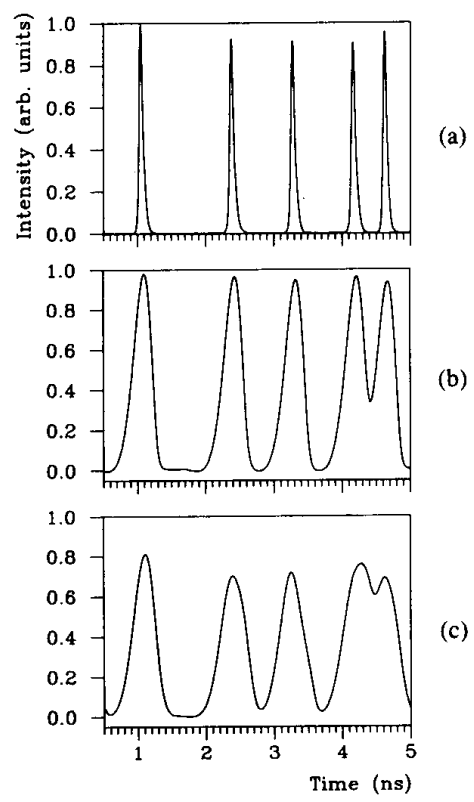

Fig. 2. Simulated responses for the TJS laser modulated at $2.24 \mathrm{Gbit} / \mathrm{s}$ : (a) the laser, (b) laser + Bessel filter, (c) laser + fiber with 0.12-ns /nm dispersion + Bessel filter. The bias current is $I_{b} \simeq I_{\text {th }}$ and the pulse current $I_{p} \simeq I_{\text {th }}$.

(a) the total light output from the TJS laser, (b) the response after filtering with a Bessel-type receiver filter, and (c) the response after a total dispersion of $\sim 0.12 \mathrm{~ns} / \mathrm{nm}$ followed by filtering.

The penalty calculated according to (8) is based on the assumption that the distribution for the sampled amplitudes of marks and spaces is Gaussian. Fig. 3 gives the probability density functions (PDF's) for the amplitudes of marks and spaces obtained from a simulation including 1400 marks and 1400 spaces. The upper skirt part for spaces and the lower skirt part for marks nearly follow the fitted Gaussian distributions which result from the mean and variance. Since these parts of the distributions are the relevant ones for calculating penalty and bit error rate, we will assume that the amplitudes for marks and spaces follow Gaussian distributions. However, it is generally not possible to reproduce the parts of the PDF with very low probability density by a numerical simulation, because it would require an enormous amount of computing time. The number of marks and spaces included in the simulations allows the $S / N$ for the transmitted signal to be estimated within $\pm 0.1 \mathrm{~dB}$.

The calculated $S / N$ and RMS pulse jitter are shown as a function of fiber dispersion in Fig. 4 for the 1300-nm laser modulated with a $8 \mathrm{~B} / 10 \mathrm{~B}$ bit sequence [13] at 565 Mbit/s. Results obtained with and without the Langevin noise terms included in the calculations are indicated by filled and open markers, respectively. Bias and pulse currents are $1.0 \cdot I_{\mathrm{th}}$ and $0.3 \cdot I_{\mathrm{th}}$, respectively, where $I_{\mathrm{th}}$ is the threshold current. For the laser itself (i.e., zero dispersion) the estimated $S / N$ is $27.6 \mathrm{~dB}$ and the jitter of the pulses is 1 percent of the clock period. The noise caused 
TABLE I

Laser Parameters USEd in the Simulations of the 2.24-GBit / $\mathrm{s}$ SYSTEM WITH A TJS LASER AND THE 565-MBIT/S SYSTEM WITH A BH LASER

\begin{tabular}{|c|c|c|c|}
\hline Parameter & & TJS & BH \\
\hline Bias current & & $1.01 \cdot 1_{1 \mathrm{~h}}$ & $I_{1 h}$ \\
\hline Pulse current + bias current & & $2.09 \cdot I_{\text {th }}$ & $1.3 \cdot \mathrm{f}_{\mathrm{th}}$ \\
\hline Duty cycle & & 0.49 & 0.40 \\
\hline Bit rate, $B_{0}$ & Gbit/s & 2.24 & 0.565 \\
\hline Gain constant, Gix & $s-1$ & $105 \cdot 104$ & $2.50 \cdot 10^{4}$ \\
\hline Nonlinear gain cónstant, $x$ & & $3.50 \cdot 10^{4}$ & $3.0 \cdot 10^{-8}$ \\
\hline Carrier number at transparcney $N_{41}$ & & $1.43 \cdot 10^{x}$ & $1.0 \cdot 10^{8}$ \\
\hline Shift of gain peak, $d t_{0} / d N$ & in & $+43+10 \cdot 17$ & $-1.21 \cdot 10 \cdot 16$ \\
\hline Wavelength fur max. gain, $A_{11}$ & nm & 887 & 1300 \\
\hline Wavelength for max spontanemus tmission & nın & 887 & 1300 \\
\hline FWIIM of gain spectrum, $\Delta h_{6}$ & $\mathrm{~nm}$ & 10 & 25 \\
\hline FWIIM of spon. emission spectrum, $\Delta l_{n}$ & $\mathrm{~nm}$ & 12 & 100 \\
\hline Internal loss, $a_{i}$ & $\mathrm{~cm}^{-1}$ & 10 & 32 \\
\hline Group index, $n_{k}$ & & 4.2 & 4.0 \\
\hline Refractive inde' $x, n_{r}$ & & 3.6 & 3.4 \\
\hline Nonradiative recombinat ion const. . A & 51 & $10^{8}$ & $10^{k}$ \\
\hline Band-to-band recomb const 13 & $\$ 1$ & 0.70 & 1.0 \\
\hline Auger recombination const. $C$ & $i^{\prime}$ & 0 & $3.0 \cdot 10^{-9}$ \\
\hline Hole number at equilibrium, $\mathrm{P}_{0}$ & & $7.4 \cdot 10^{8}$ & 0 \\
\hline Confinement factor, $\mathrm{I}$ & & 0.5 & 0.3 \\
\hline Cavity length, l & $\mu \mathrm{m}$ & 286 & 250 \\
\hline Width of active region, w & $\mu \mathrm{m}$ & 2.5 & 2.0 \\
\hline Thickness of active layer, d & $\mu \mathrm{m}$ & 0.2 & 0.2 \\
\hline Carrier lifetime at threshold, $\iota_{\text {s }}$ & ns & 1.3 & 2.64 \\
\hline Photon lifetime, 1 & ps & 2.80 & 1.65 \\
\hline Relaxation frequency, $i_{\text {rers }}$ & Gil\%. & 4.81 & 1.98 \\
\hline Threshold current, $l_{\mathrm{th}}$ & $\mathrm{m} \Lambda$ & 25.9 & 11.0 \\
\hline
\end{tabular}

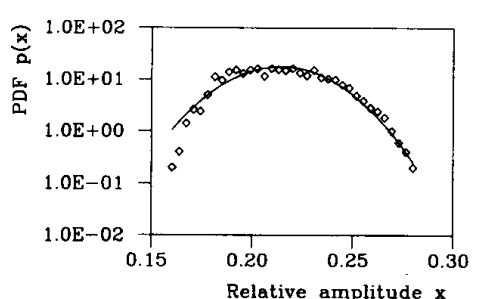

(a)

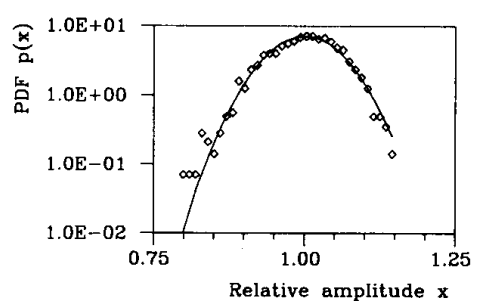

(b)

Fig. 3. Probability density function for the amplitude of (a) spaces and (b) marks after $0.2 \mathrm{~ns} / \mathrm{nm}$ of dispersion. The plots are based on a bit sequence with 1400 marks and 1400 spaces. The solid curves give the fitted Gaussian distributions.

by intersymbol interference corresponds to a $S / N$ of 36 $\mathrm{dB}$ for the laser itself which is $\sim 7 \mathrm{~dB}$ higher than the results obtained when the Langevin noise terms are in-

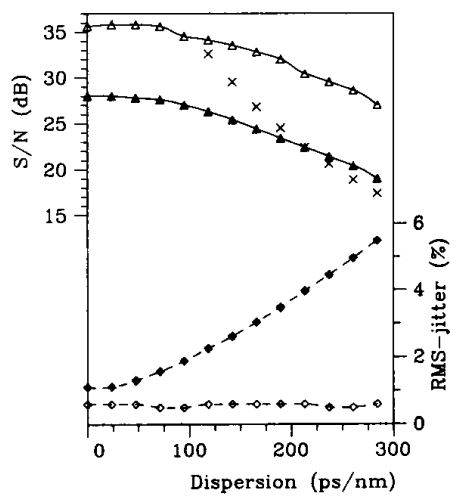

Fig. 4. Simulated $S / N$ and RMS-jitter (relative to a clock period) versus dispersion for a $1300-\mathrm{nm}$ BH laser modulated with a coded bit sequence at $565 \mathrm{Mbit} / \mathrm{s}$. The filled and open markers give the results with and without the Langevin noise terms included in the calculations, respectively. The $S / N$ predicted by (12) is also shown (x).

cluded. As the dispersion increases the $S / N$ decreases due to the mode partition noise and the jitter is correlated with the $S / N$ as would be expected. A $S / N$ of $20 \mathrm{~dB}$ (giving a $1-\mathrm{dB}$ power penalty) results for a dispersion of 260 $\mathrm{ps} / \mathrm{nm}$. This corresponds to a relative dispersion (per clock period) $D_{\text {rel }}$ of:

$$
D_{\text {rel }}=\Delta \lambda \cdot \delta \cdot L \cdot B_{0}=0.37
$$


where $\Delta \lambda$ is the FWHM of the time averaged spectrum, $\delta$ is the dispersion coefficient, and $B_{0}$ is the bit rate. This can be compared to the relative dispersion of 0.35 which is given in [14] as the upper limit. The same limit of 0.35 is extracted from the experimental results for a 405 Mbit/s system reported in [15]. Experimental results by Bridge et al. for a 565-Mbit/s NRZ system resulted in a maximum allowable relative dispersion of 0.28 [16]. From this comparison we conclude that the numerical model presented here is able to predict the dispersion limits with reasonable accuracy. But it should be kept in mind that the practical lasers may have a more irregular spectrum than predicted by the model and consequently lower dispersion limits can be encountered in practical systems. At the dispersion resulting in a $S / N$ of $20 \mathrm{~dB}$ the RMS pulse jitter is 5 percent of a clock period. Also indicated in the diagram are the values for $S / N$ which result from Ogawa's analytical expression (12) taking the relative mode intensities and the calculated $k$ of 0.30 as input parameters (the $k$ value results from (10)). From the results based on (12) we find an allowed dispersion of $D_{\text {rel }}=0.35$ as can be seen from Fig. 4 and we can conclude that the equation gives reasonable results for high dispersion where the partition noise is dominating. For low dispersion the $S / N$ due to intensity noise in the laser is the dominant noise source.

Similarly, Fig. 5 gives the calculated $S / N$ and RMS jitter versus dispersion for the TJS laser modulated at 2.24 Gbit/s with a coded bit sequence. The bias and pulse currents are $1.01 \cdot I_{\text {th }}$ and $1.08 \cdot I_{\text {th }}$, respectively. Results obtained with the Langevin noise terms included in the calculation are given as filled markers and show that the $S / N$ for the output signal from the laser is $31.9 \mathrm{~dB}$ and the jitter is $\sim 1$ percent of a clock period. Similar calculations without the Langevin noise terms included result in a higher $S / N$ and a smaller jitter as indicated by the open markers. For low dispersion the intersymbol interference gives a significant contribution to both the $S / N$ and RMS-jitter. Again the $S / N$ is decreasing with the dispersion and $S / N=20 \mathrm{~dB}$ is obtained for a dispersion of $120 \mathrm{ps} / \mathrm{nm}$ which corresponds to a relative dispersion per clock period of

$$
D_{\text {rel }}=\Delta \lambda \cdot \delta \cdot L \cdot B_{0}=0.44 \text {. }
$$

This limit corresponds to $\sim 1.5 \mathrm{~km}$ of fiber at $880-\mathrm{nm}$ wavelength or a (bit rate) - (link length) product of 3.6 Gbit $/ \mathrm{s} \cdot \mathrm{km}$. The corresponding RMS pulse jitter is 5.1 percent of a clock period. Again the values of $S / N$ which result from Ogawa's analytical expression (12) are indicated taking the relative mode intensities (see Fig. 6(a)) and the calculated $k$ of 0.32 as input parameters. These data give an allowed dispersion of $D_{\text {rel }}=0.51$ and also in this case there is an acceptable agreement for high dispersion between our simulated results and those resulting from (12).

Based on the responses of the individual longitudinal modes to the marks in the bit sequence it is possible to obtain a time averaged spectrum and the $k$-factors can be

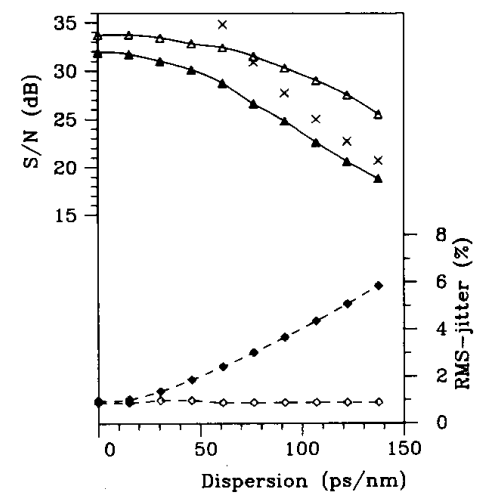

Fig. 5. Simulated $S / N$ and RMS-jitter versus dispersion for an 880-nm TJS laser modulated with a coded bit sequence at $2.24 \mathrm{Gbit} / \mathrm{s}$. The filled and open markers give the results with and without the Langevin noise terms included in the calculations, respectively. The $S / N$ predicted by (15) is also shown $(x)$.

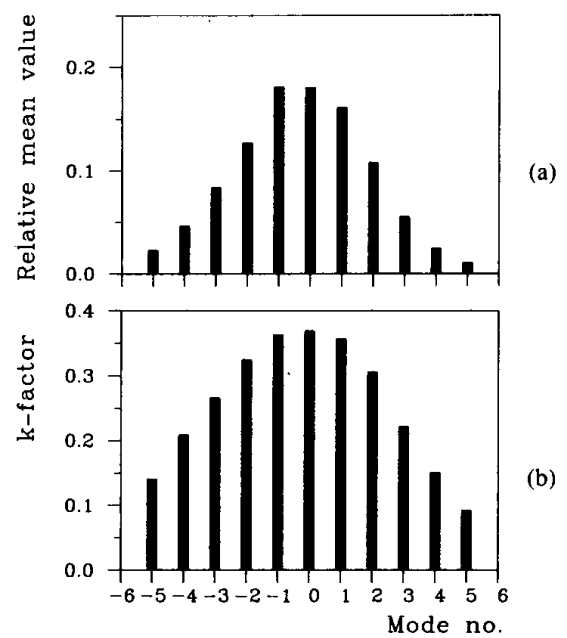

Fig. 6. (a) Relative mean intensities and (b) $k$-factors for the lasing modes in the 2.24-Gbit/s simulation for which results are given in Fig. 5 .

TABLE II

Allowed Relative Dispersion Per Clock Period $D_{\text {rel }}$ For Different Bit Rates $B$. The Simulations ARE FOR AN 880-NM TJS LASER AND THE NoRMalized PULSE CURRENTS $I_{p} / I_{\text {th }}$ USED IN THE Simulations aRe also GIVEN TOGETHER WITH THE RESULTING RESONANCE FREQUENCY

\begin{tabular}{|c|c|c|c|}
\hline \hline B, Gbit/s & $D_{\text {rel }}$ & $f_{\text {res }}, \mathrm{GHz}$ & $\mathrm{I}_{\mathrm{p}} \mathrm{I}_{\mathrm{th}}$ \\
\hline .565 & 0.39 & 2.1 & 0.200 \\
\hline 1.12 & 0.40 & 3.3 & 0.492 \\
\hline 2.24 & 0.44 & 4.9 & 1.086 \\
\hline 4.5 & 0.44 & 6.6 & 1.992 \\
\hline
\end{tabular}

derived from (11). Results corresponding to the conditions for Fig. 5 are shown in Fig. 6. We observe that the $k$ factors for the dominating modes range from 0.3 to 0.4 and that $k$ is decreasing with the distance between the mode under consideration and the central mode. The $k$ factors are in reasonable agreement with those reported in the literature [16], [17].

We investigated the allowed relative dispersion $D_{\text {rel }}$ for bit rates between $565 \mathrm{Mbit} / \mathrm{s}$ and $4.5 \mathrm{Gbit} / \mathrm{s}$ for both the $880-\mathrm{nm}$ TJS laser and the $1300-\mathrm{nm}$ BH laser. The results 
TABLE III

Allowed Relative Dispersion Per Clock Period $D_{\text {rel }}$ For Different Bit RATES $B$. The Simulations aRe For a 1300 -NM BH LaSER AND THE Normalized PUlse CuRRENTS $I_{p} / I_{\text {th }}$ USED IN THE SimUlations aRE ALSO GIVEN TOGETHER WITH THE RESUlting RESONANCE FREQUENCY

\begin{tabular}{|c|c|c|c|}
\hline \hline B, Gbit/s & $D_{\text {rel }}$ & $f_{\text {res }}, \mathrm{GHz}$ & $\mathrm{I}_{\mathrm{p}} / \mathrm{I}_{\text {th }}$ \\
\hline .565 & 0.37 & 2.0 & 0.300 \\
\hline 1.12 & 0.39 & 3.2 & 0.792 \\
\hline 2.24 & 0.41 & 4.8 & 1.792 \\
\hline 4.5 & 0.40 & 6.2 & 2.992 \\
\hline
\end{tabular}

are given in Table II and Table III together with the pulse currents which were used in the simulations and the corresponding resonance frequency of the laser. As seen a relative dispersion of $\sim 0.4$ can be tolerated independently of the bit rate as long as the resonance frequency is higher than the bit rate.

\section{Experimental Results}

\section{A. Experimental Technique}

The first task has been to establish experimental procedures for measurement of the signal-to-noise ratio for links which are operated under realistic conditions. A schematic diagram of the experimental set-up is shown in Fig. 7. The lasers are dc-coupled and pulse modulated with pulse currents of typically $0.5-1.0$ times the threshold current. In most of the investigations we used an Anritsu word generator with an upper bit rate of 2.5-Gbit $/ \mathrm{s}$ RZ. In all our measurements the lasers were modulated in order to simulate the conditions in PCM systems as closely as possible. With this setup three kinds of investigations can be performed: 1) measurement of reflection noise by direct feedback from a mirror, 2) link simulations with different configurations of multimode (MM) and single mode (SM) fiber, and 3) analysis of the spectral properties of the laser by a spectrometer.

The light is detected by a fast $p-i-n$ photodiode followed by a broadband amplifier $(20 \mathrm{~dB}$ in the passband of $10-4200 \mathrm{MHz}$ ). The signal is then filtered by a lowpass filter with a 3-dB bandwidth of $\sim 1 \mathrm{GHz}$. The amplitudes of marks and spaces are sampled with a sampling oscilloscope, digitized and dumped to a PC for calculations of variance, mean value, $S / N$, and distribution of the pulse amplitude. Mean values $b_{j}$ and variances $\sigma_{j}^{2}$ for the sampled amplitudes $S_{j k}$ of the marks $(j=1)$ and the spaces $(j=0)$ are found:

$$
\begin{aligned}
b_{j} & =\frac{1}{N} \sum_{k=1}^{N} S_{j k} \\
\sigma_{j}^{2} & =\left(\frac{1}{N} \sum_{k=1}^{N} S_{j k}^{2}\right)-b_{j}^{2} .
\end{aligned}
$$

The $S / N$ is then calculated according to (7). Before the $S / N$ is calculated it is, however, necessary to correct for the noise of the detection system. The procedure is to block off the light to the detector and then measure the noise $\sigma_{\text {det }}^{2}$ which is uncorrelated with the noise from the

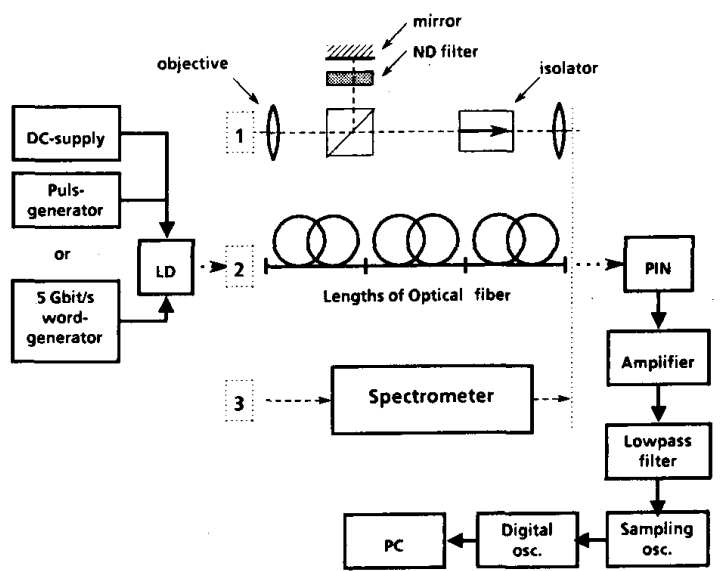

Fig. 7. Experimental setup.

laser. The corrected variances $\sigma_{j \text {,cor }}^{2}$ for marks or spaces are then given by:

$$
\sigma_{j, \text { cor }}^{2}=\sigma_{j}^{2}-\sigma_{\mathrm{det}}^{2} .
$$

We have assumed the detector shot noise to be negligible (which can be verified by using a white noise light source [18]).

The evaluation of the $S / N$ is for most of our measurements based on 2046 sampled values for marks and spaces, respectively, and the resulting measurement uncertainty is smaller than 10 percent.

\section{B. Results}

The results presented here are all for a Mitsubishi TJS laser module emitting at $880-\mathrm{nm}$ wavelength. Fig. 8 gives the relative mean intensities and $k$ factors resulting from sampled measurements of the pulse responses for the individual longitudinal modes. The spectra are obtained for bias currents of 24 and $27 \mathrm{~mA}\left(I_{\mathrm{th}}=26 \mathrm{~mA}\right)$ while the laser is modulated at $2.24 \mathrm{Gbit} / \mathrm{s}$ ( 50 -percent duty cycle) with a pulse amplitude of $28 \mathrm{~mA}$. The detection bandwidth of $1 \mathrm{GHz}$ is determined by the low-pass filter as discussed above. As predicted by our numerical model (c.f., Fig. 6(b)) the $k$ values are increasing with the mean amplitude of the modes. This behavior was also reported in [15]. The increase of the $k$ factors with the bias current is attributed to the fact that the spectrum becomes narrower.

We investigated the $S / N$ measured for the TJS laser under feedback. As shown in Fig. 7 part of the light is reflected back into the laser using a beamsplitter and a mirror. The laser is modulated with the repetitive bit sequence $\cdots 100 \cdots$ at $2.24 \mathrm{Gbit} / \mathrm{s}$. The external cavity length is $20 \mathrm{~cm}$ resulting in a round-trip time of $1.33 \mathrm{~ns}$ which equals the time between two adjacent marks. Maximum feedback is obtained by adjusting the mirror for maximum optical mean output power from the laser.

Fig. 9 shows the light-current characteristics with maximum feedback and without feedback. From the decrease in threshold current $\Delta I_{\text {th }}$ it is possible to estimate the feedback level $\kappa$ if we assume single mode operation, weak 
Relative mean value
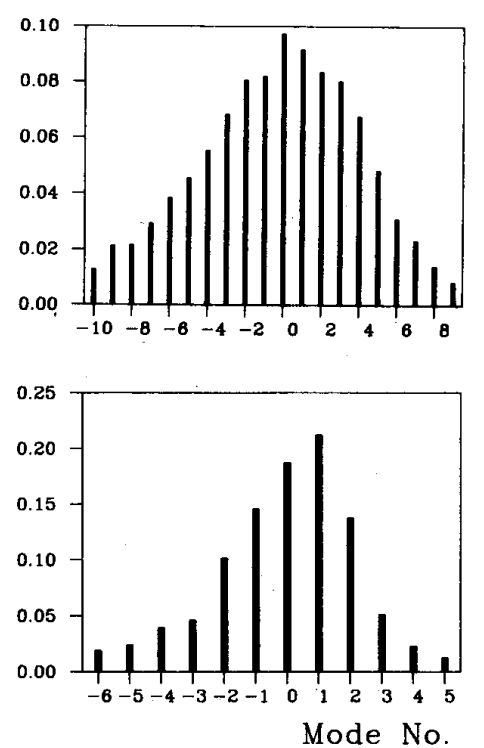

$k$-factor
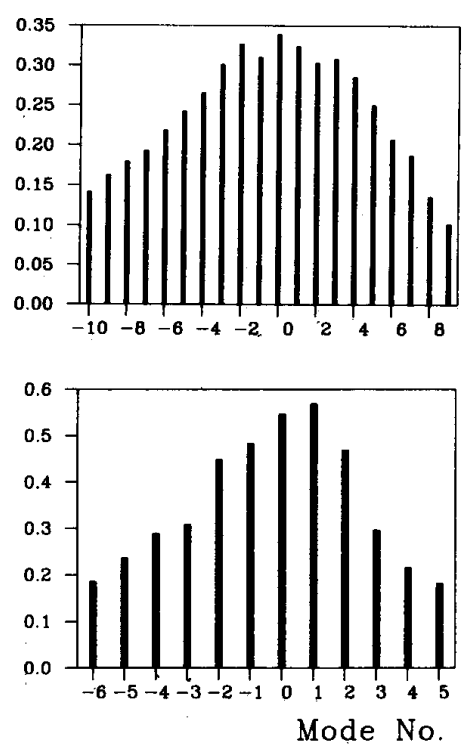

$\mathrm{I}_{\mathrm{b}}=$

$24 \mathrm{~mA}$

$27 \mathrm{~mA}$

Fig. 8. Measured relative mean intensities and $k$-factors for the longitudinal modes in the TJS laser. Bias currents are $I_{b}=24 \mathrm{~mA}$ and $I_{b}=27$ $\mathrm{mA}$ and the pulse current is $I_{p}=28 \mathrm{~mA}$.

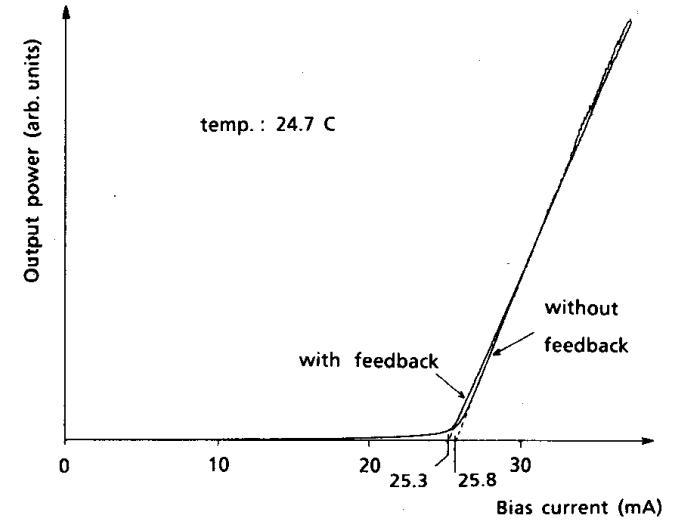

Fig. 9. Light-current characteristics for the TJS laser with maximum feedback and without feedback.

feedback, and a long external cavity [19]

$$
\kappa \simeq \frac{\tau_{s, \mathrm{dyn}}}{\tau_{s, \text { stat }}} \frac{\Gamma G_{N} N_{\mathrm{th}, 0} l}{v_{g}} \frac{\Delta I_{\mathrm{th}}}{I_{\mathrm{th}, 0}}=\frac{\tau_{s, \mathrm{dyn}}}{\tau_{s, \mathrm{stat}}} c_{1} \frac{\Delta I_{\mathrm{th}}}{I_{\mathrm{th}, 0}} .
$$

Here, $\tau_{s, \text { stat }}=\left[R\left(N_{\mathrm{th}, 0}\right) / N_{\mathrm{th}, 0}\right]^{-1}$ and $\tau_{s, \text { dyn }}=$ $[d R / d N]^{-1}$ are the static and dynamic carrier lifetimes, which will be different unless a linear recombination model is used. $N_{\text {th }, 0}$ and $I_{\text {th, } 0}$ are the threshold carrier density and threshold current for the laser without feedback, $v_{g}$ is the group velocity, $l$ is the diode cavity length and the other parameters are described in Section II. For the TJS laser (c.f., Table I) the proportionality constant $c_{1}$ in (13) is found to be 4.43 and $\tau_{s, \text { dyn }} / \tau_{s, \text { stat }}=(1.093$ ns $) /(1.303 \mathrm{~ns})=0.84$. Consequently for the TJS laser under investigation we estimated a maximum feedback level of $20 \log _{10}(\kappa)=-23 \mathrm{~dB}$.

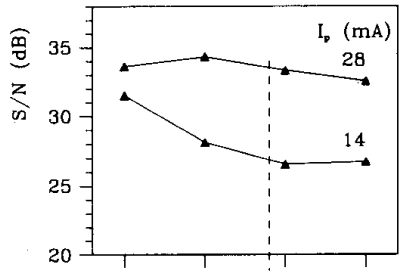

(a)

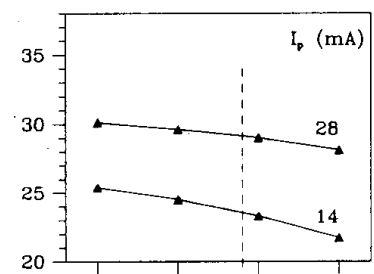

(b)

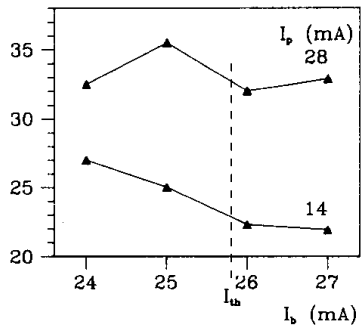

(c)

Fig. 10. $S / N$ ratio versus bias current $I_{b}$ with the pulse current $I_{p}$ as a parameter for: (a) the solitary laser, (b) feedback level of $-23 \mathrm{~dB}$, (c) feedback from a SM fiber pigtail.

Fig. 10(a)-(c) gives the measured $S / N$ versus bias current $I_{b}$ with the pulse current $I_{p}$ as a parameter for a) the solitary laser, b) a feedback level of $-23 \mathrm{~dB}$, and c) feedback from a 1.5 -m-long tapered lens-ended fiber pigtail. The $S / N$ increases with the pulse current which may be 
attributed to the increase in the signal level for marks and a decrease of the noise level due to increasing relaxation frequency of the laser. For strong feedback (Fig. 10(b)) the $S / N$ is $3-4$ dB lower than for the solitary laser (Fig. $10(\mathrm{a}))$. This is not considered to be a serious decrease of the $S / N$ compared to the $12-\mathrm{dB}$ decrease which we have observed for similar experiments with DFB lasers. From measurements with a microwave spectrum analyzer it was noticed that the noise spectrum was shifting between two levels indicating that the noise level is very dependent on the phase of the reflected light.

For the solitary laser the $S / N$ is $-33 \mathrm{~dB}$ for $I_{b}=26$ $\mathrm{mA}$ and $I_{p}=28 \mathrm{~mA}$. Our numerical model resulted in an $S / N$ of $31.9 \mathrm{~dB}$ (Fig. 5). Considering the uncertainties in the estimated laser parameters and the differences between the simulated and the experimental characteristics for the receiver filter, there is an acceptable agreement with our experimental result.

The influence of reflections from a 1.5-m-long SM fiber pigtail is also investigated since the pigtail may cause the most serious feedback to the laser in a real system. A lensended taper was formed on the fiber to ensure good coupling between laser and fiber as well as low feedback to the laser from the near end of the fiber (estimated lower than $-50 \mathrm{~dB}[20])$. The feedback from the far end of the pigtail is estimated to $-34 \mathrm{~dB}$ assuming that the coupling efficiency from the fiber to the laser cavity equals that from the laser to the fiber [20]. Compared to the solitary laser the $S / N$ ratio for $I_{p}=14 \mathrm{~mA}$ is up to $7 \mathrm{~dB}$ lower with feedback from the pigtail. For $I_{p}=28 \mathrm{~mA}$ there is only a difference of $1 \mathrm{~dB}$ between the two cases.

The $S / N$ was also investigated for two link configurations employing single mode (SM) fiber. The laser diode is again modulated with the $\cdots 100 \cdots$ bit sequence at the bit rate $2.24 \mathrm{Gbit} / \mathrm{s}$ (RZ) and the detection bandwidth is $1 \mathrm{GHz}$ as described above. Fig. 11 gives the $S / N$ versus bias current with the pulse current as a parameter for a 1000-m-long SM fiber with a tapered input end. The feedback from the far end of the fiber is weak so the $S / N$ is mainly contributed by the partition noise. For $I_{p}=28$ $\mathrm{mA}$ the decrease due to mode partition noise is $\sim 8 \mathrm{~dB}$ compared to the $S / N$ for the laser itself (Fig. 10(a)). As expected the $S / N$ increases with the bias current due to decreasing spectral width (see Fig. 8). Also shown is the $S / N$ calculated from Ogawa's equation (12) for $I_{p}=28$ $\mathrm{mA}$ and taking a chromatic dispersion of $75 \mathrm{ps} / \mathrm{nm}$. The calculated $S / N$ values agree with the measured values within $2 \mathrm{~dB}$, which is acceptable considering the experimental uncertainties involved and the approximations in Ogawa's theory. As also shown in Fig. 11 for a bias of $26 \mathrm{~mA}$ our numerical model resulted in $S / N$ ratios of 28.8 and $26.5 \mathrm{~dB}$ for a $\cdots 1010 \cdots$ bit sequence and a coded bit sequence, respectively. These values are up to $4 \mathrm{~dB}$ higher than the measured value and the discrepancy may partly be accounted for by the calculated $k$ factor of 0.34 compared to the measured value of 0.43 . Again it should be mentioned that our theoretical model does not take into account the influence of optical feedback. Feedback, however should be of little importance in the experiment.

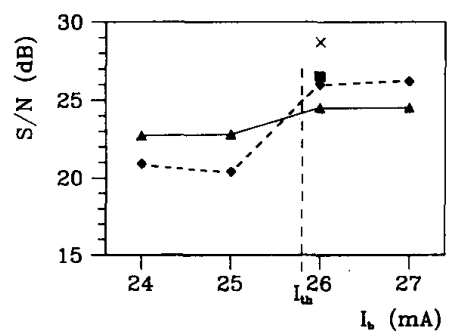

Fig. 11. $S / N$ versus bias current for transmission over $1000 \mathrm{~m}$ of $\mathrm{SM}$ fiber. Experimental results are shown as triangles ( $\Delta)$. Also shown in the $S / N$ predicted by (12) using the experimental $k$-values ( $\bullet$ ) and the $S / N$ predicted by our numerical model for a coded bit sequence (a) and for a $\cdots 1010 \cdots$ sequence $(x)$. The bit rate is $2.24 \mathrm{Gbit} / \mathrm{s}$ and the pulse current is $28 \mathrm{~mA}$.

\section{Summary}

A numerical model based on multimode rate equations for Fabry-Perot type semiconductor lasers was established for the purpose of predicting the noise due to the laser in fiber-optic links. The model can account for the laser intensity noise and for the mode partition noise which after transmission over dispersive fibers causes excess noise at the receiver. It was found that the relative dispersion per clock period which is the product between the dispersion, the fiber length, the spectral halfwidth of the laser, and the bit rate, must be lower than 0.37 to ensure power penalties due to mode partitioning lower than $1 \mathrm{~dB}$. The allowed relative dispersion was found to be almost independent of the simulated bit rate in the investigated range from $565 \mathrm{Mbit} / \mathrm{s}$ to $4.5 \mathrm{Gbit} / \mathrm{s}$. Pattern effect due to pseudorandom codes gives a noise-like probability density function. The noise due to the pattern effect can be significant for short lengths of fiber, but for larger dispersion the mode partition noise is dominating. Experimentally we investigated the $S / N$ ratio for an 880 -nm TJS laser modulated at $2.2 \mathrm{Gbit} / \mathrm{s}$. For the solitary laser a $S / N$ of $33 \mathrm{~dB}$ resulted in good agreement with an estimated value of $32 \mathrm{~dB}$ from the numerical model. Strong feedback to the laser degraded the $S / N$ by $3-4 \mathrm{~dB}$, which is significantly less than the degradation observed for DFB lasers. Transmission over $1000 \mathrm{~m}$ of single mode fiber degraded the $S / N$ by $8 \mathrm{~dB}$ compared to the solitary laser. For comparison our numerically estimated $S / N$ was $2-4$ $\mathrm{dB}$ higher than the measured value. In summary, we found that the model is a good tool for understanding the behavior of semiconductor lasers under fast modulation. Considering experimental uncertainties we found the simulated results to be in good agreement with experimental results.

\section{ACKNOWLEDGMENT}

The authors are indebted to Dr. J. Crow and Dr. R. Bates for help and advice.

\section{REFERENCES}

[1] K. Ogawa and R. S. Vodhanel, "Measurements of mode partition noise of laser diodes," IEEE J. Quantum Electron., vol. QE-18, pp. 1090-1093, July 1982.

[2] Y. Okano, K. Nakagawa, and T. Ito, "Laser mode partition noise 
evaluation for optical fiber transmission," IEEE Trans. Commun., vol. COM-28, pp. 238-244, Feb. 1980.

[3] N. H. Jensen, H. Olesen, and K. E. Stubkjaer, "Partition noise in semiconductor lasers under $\mathrm{CW}$ and puised operation," IEEE $J$. Quantum Electron., vol. QE-23, pp. 71-80, Jan. 1987.

[4] R. A. Linke, "Transient chirping in single-frequency lasers: Lightwave systems consequences," Electron Lett., vol. 20, pp. 472 474, May 1984.

[5] N. A. Olsson, W. T. Tsang, H. Temkin, N. K. Dutta, and R. Á. Logan, "Bit-error-rate saturation due to mode-partition noise induced by optical feedback in $1.5-\mu \mathrm{m}$ single longitudinal-mode $\mathrm{C}^{3}$ and DFB semiconductor lasers," J. Lightwave Technol., vol. LT-3, pp. 215218, Apr. 1985

[6] H. Kuwahara, H. İmai, and M. Sasaki, "Intensity noise of InGaAsP / InP lasers under the influence of reflection and modulation," Opt. Commun., vol. 46, pp. 315-322, July 1983.

[7] J. D. Crow, IBM Research Center, Yorktown Heights, NY, private communication.

[8] G. Arnold and K. Petermann, "Intrinsic noise of semiconductor lasers in optical communication systems," Opt. Quantum Electron., vol. 12, pp. 207-219, May 1980.

[9] J. E. Bowers, T. L. Koch, B. R. Hemenway, D. P. Wilt, T. J. Bridges, and E. G. Burkhardt, "High-frequency modulation of $1.52-\mu \mathrm{m}$ vapor-phase-transported InGaAsP lasers," Electron Lett., vol. 21, pp. 297-299, Mar. 1985.

[10] R. Olshansky, C. B. Su, J. Manning, and W. Powazinik, "Measurements of radiative and nonradiative recombination rates in InGaAsP and GaAlAs light sources, 'IEEE J. Quantum Electron., vol. QE-20, pp. 838-854, Aug. 1984.

[11] S. D. Personick, "Receiver design for digital fiber optic communication systems, Parts I and II," Bell Sysi. Tech. J., vol. 52, pp. 843886, July-Aug. 1973

[12] K. Ogawa, "Analysis of mode partition noise in laser transmission systems,' IEEE J. Quantum Electron., vol. QE-18, pp. 849-855 May 1982.

[13] A. X. Widmer and P. A. Franaszek, "A DC-balanced partitioned block 8B/10B transmission code," IBM J. Res. Develop., vol. 27, pp. 440-451, Sept. 1983.

[14] E. Iwahashi, "Trends in long wavelength single-mode transmission systems and demonstrations in Japan," private communications, 1981.

[15] M. Mizuguchi, T. Ogawa, and K. Kameo, "405 Mbit/s single-mode optical fiber transmission systems,'” Fujitsu Sci. Tech. J., vol. 21 , pp. 50-66, Mar. 1985.

[16] P. Bridge and G. G. Windus, "Laser diode spectral degradations in $565 \mathrm{Mbit} / \mathrm{s}$ single mode optical systems," private communication.

[17] K. Mathyssek and E. Hörmann, "Parameter dependence on the mode partition noise and a new method for its evaluation," in Proc. IOOC. ECOC '85 (Venezia, Italy), Oct. 1985, vol. I, pp. 765-768.

[18] T. L. Paoli, "Noise characteristics of stripe-geometry double-heterostructure junction lasers operating continuously-I. Intensity noise at room temperature," IEEE J. Quantum Electron., vol. QE-11, pp 276-283, June 1975.

[19] H. Olesen et al., unpublished results.

[20] H. Kuwahara, Y. Onoda, M. Goto, and T. Nakagami, "Reflected light in the coupling of semiconductor lasers with tapered hemispherical end fibers."' Appl. Opt., vol. 22, pp. 2732-2738, Sept. 1983.

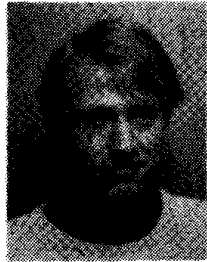

C. Michael Olsen was born in Glostrup, Denmark, on May 25, 1961. In 1986 he received the M.Sc. degree in electrical engineering from the Electromagnetics Institute, Technical University of Denmark.

From 1986 to 1987 he worked as an Associate Researcher at the Electromagnetics Institute, and since 1988 he has been persuing his Industrial Ph.D. degree at NKT Elektronik, Glostrup, Denmark. The topic of his investigations is high-speed modulation and system performance of semiconductor lasers. In the summer of 1987 he was a Guest Researcher at the IBM T. J. Watson Research Center, Yorktown Heights, NY, and since July 1988 he has been a Visiting Scientist at Bellcore, Morristown, NJ.

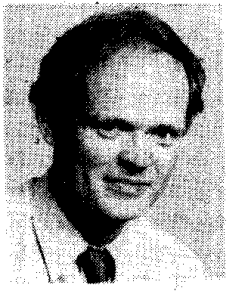

Kristian E. Stubkjaer (S'76-M'81) was born in Arhus, Denmark, in 1953. He received the M.Sc. and Ph.D. degrees from the Technical University of Denmark, Copenhagen, in 1977 and 1981, respectively.

From 1979 to 1981 he studied at the Tokyo Institute of Technology, Tokyo, Japan, with a scholarship from the Japanese Government. From 1981 to 1982 he was drafted for military service at the Danish Defence Research Establishment in Copenhagen. From 1982 to 1983 he was a Visiting Scientist at the IBM T. J. Watson Research Center, Yorktown Heights, $\mathrm{NY} . \mathrm{He}$ is now an Associate Professor at the Electromagnetics Institute, Technical University of Denmark, Lyngby, Denmark, where he is working in the field of optical communication. Since June 1985 he has been Director of the Electromagnetics Institute.

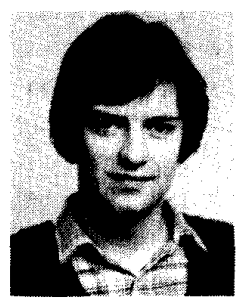

Henning Olesen was born in Vinderup, Denmark, on October 11, 1955. He received the M.Sc. and $\mathrm{Ph} . \mathrm{D}$. degrees in electrical engineering from the Technical University of Denmark, Lyngby, Denmark, in 1980 and 1982, respectively.

From 1982 to 1984 he was a Postdoctoral Re search Associate at the Electromagnetics Institute, Technical University of Denmark, where he worked on coherent optical transmission systems. In 1983 he stayed as a Visiting Scientist at Musashino ECL, Nippon Telegraph and Telephone Corporation, Tokyo, Japan. Since 1984 he has been employed at TFL Telecommunications Research Laboratory, $\mathrm{H} \phi$ rsholm, Denmark. His primary research interests are the characteristics of semiconductor lasers for coher ent systems, in particular multielectrode DFB and DBR lasers and lasers with optical feedback. 